\title{
Hybrid Optimization Process Applied to Tuning of Dynamic Matrix Control: Study Case with DC Motor
}

\author{
Douglas Freire de Carvalho, Cleber Asmar Ganzaroli, Luiz Alberto do Couto, Rafael Nunes \\ Hidalgo Monteiro Dias, Wesley Pacheco Calixto
}

\begin{abstract}
This paper presents study about Dynamic Matrix Control (DMC) controller applied to speed control of DC motor. DMC controller parameters (prediction horizon, control horizon and damping rate of reference) are obtained through optimization methods employing heuristic, deterministic and hybrid strategies. The use of advanced control technique combined with using of optimization methods aims to achieve highly efficient control, reducing the transient state period and variations in steady state. These methods were applied on a simulation model in order to verify which one provides better control results.
\end{abstract}

Index Terms - Predictive Control, Deterministic Optimization, Heuristic Optimization, Hybrid Optimization, DC motor.

\section{INTRODUCTION}

$\mathrm{D}$ IRECT current (DC) motors are used in various situations ranging from residential applications to purposes of industrial scale. Utilization of DC motors implies, often, in its speed control. Aiming to do a high quality speed control of DC machines several number of techniques has been developed [1].

Control systems techniques are employed seeking to promote proper implementation of processes, generally, controlling manipulated system variables to obtain desired values for output system variables [2]. Control systems techniques are often applied to control speed of DC machines aiming to promote proper implementation of processes.

Model based Predictive Control (MPC) refers to determinate class of control algorithms that seek to obtain optimal control signal minimizing certain objective function, explicitly using process model. By calculating series of actions of manipulated variables, the MPC seeks, overall, that the system output reaches its reference trajectory. MPC have been developed seeking to solve problems of process control in industrial environment, particularly in oil industry, being initially proposed by Richalet at 1978, proposing the Model Predictive Heuristic Control (MPHC) and by Cutler \& Remaker at 1980, proposing the Dynamic Matrix Control

Authors thank the National Counsel of Technological and Scientific Development $(\mathrm{CNPq})$, the Research Support Foundation for the State of Goiás (FAPEG) and the Coordination for the Improvement of Higher Education Personnel (CAPES) for financial assistance to this research.
(DMC) [3]. MPC are employed in various areas being widely accepted by industry and academia, applicable to various systems (multivariable, nonlinear, with high dead time, constrained variables, etc.).

Currently DMC is the most popular MPC algorithm, widely used for control of chemical processes and with good results in several other applications. DMC become widely popular in industry for present intuitive operation and provide significant results [4]. DMC is based on use of step response finite model of the system to be controlled and its control strategy is presented in time domain, being more intuitive than systems modeled in state space. Applications of DMC controller for linear systems without restrictions present analytical solutions for objective function minimization problem, reducing computational costs. However, nonlinear systems or with dead time and imposition of restrictions to system are met clearly and efficiently by DMC controller [5].

Given the complexity of some problems and the search for efficient and robust controllers, controllers' optimization is presented as interesting proposal. Implementation of controllers' optimization process, generally, seeks to define optimized values for controller variables, aiming to reduce the error between reference proposed and output of controlled system [6].

The literature presents various optimization methods that can be divided into two distinct groups: i) deterministic and ii) heuristic methods. Deterministic methods follow fixed sequence from a defined starting point, returning always the same output value if the starting point is maintained. Heuristic methods seek optimal solution promoting stochastic variations from possible solutions sets, this strategy results in unpredictable sequences and return different optimal values each run, even keeping the initial conditions [6].

Both optimization methods, deterministic and heuristic, present advantages, being not possible determinate the best method for all cases. In general, heuristic methods present better results when looking for solutions in large search spaces. Deterministic methods present better performance in smaller search spaces, close to starting point.

Aiming to combine features of different methods, hybrid optimization methods are implemented seeking for better solutions that any of individuals methods could present. Hybrid optimization methods combines two or more other

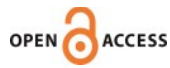

TRANSACTIONS ON ENVIRONMENT AND ELECTRICAL ENGINEERING ISSN 2450-5730 Vol 2, No 2 (2017) (C) Douglas Freire de Carvalho, Cleber Asmar Ganzaroli, Luiz Alberto do Couto, Rafael Nunes Hidalgo Monteiro Dias, Wesley Pacheco Calixto 
optimization methods to solve problems. Hybrid methods could either choosing one method depending on the data, or switching between them over the course of algorithm. The hybrid optimization methods combining heuristic and deterministic methods are widely presented in literature. In general, these hybrid methods combines the amplitude and reliability of heuristic methods with accuracy of deterministic methods [9].

\section{Methodology}

\section{A. Modeling and simulation of direct current motors}

The DC motor has mathematical models known in the literature, [8]. Generally, models are composed of two parts: the electrical and mechanical. In equating of electrical part, the parameters are resistance $\mathrm{Ra}$ and inductance $\mathrm{La}$ of armature. In equating of mechanical part, we have the moment of inertia $\mathrm{J}$ and viscous friction coefficient $\mathrm{B}$. The relation between the two parties is realized through constants of torque $\mathrm{Kt}$ and back EMF $\mathrm{Kb}$. The mathematical model of $\mathrm{DC}$ motor can be represented, in frequency domain, by the block diagram of Fig. 1.

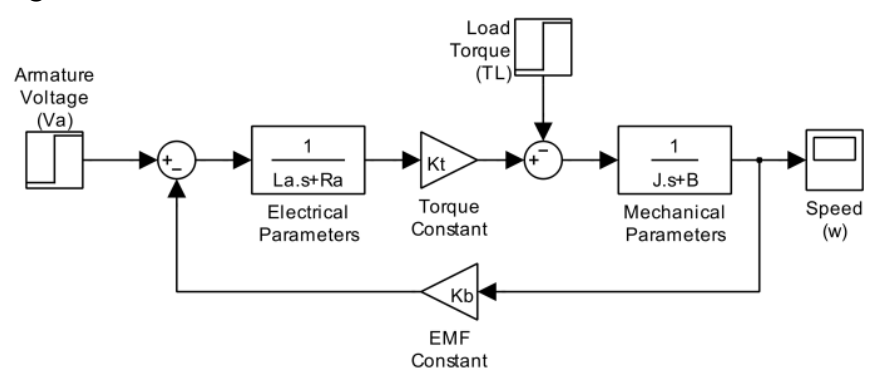

Figure 1. DC motor block diagram

Based on diagram shown in Fig. 1 and on the multiple systems reduction theory, became possible to obtain two transfer functions. In (1) the speed $\omega$ against the armature voltage $\mathrm{Va}$ and in (2) the speed $\omega$ against the load torque TL.

$$
\begin{aligned}
& \frac{\omega(s)}{V_{a}(s)}=\frac{K_{t}}{\alpha s^{2}+(\beta+\gamma) s+\delta+K_{t} K_{b}} \\
& \frac{\omega(s)}{T_{L}(s)}=\frac{-L_{a} \cdot s-R_{a}}{\alpha s^{2}+(\beta+\gamma) s+\delta+K_{t} K_{b}}
\end{aligned}
$$

where: $\alpha=\mathrm{La} \cdot \mathrm{J}, \beta=\mathrm{La} \cdot \mathrm{B}, \gamma=\mathrm{Ra} \cdot \mathrm{J}$ e $\delta=\mathrm{Ra} \cdot \mathrm{B}$.

\section{B. Dynamic Matrix Control}

Dynamic Matrix Control (DMC) controller enables inclusion of restrictions imposed by system to its basic structure making its application more efficient. The inclusion of restrictions in control law enable the DMC controller to perform actions on the system boundary, really close the restrictions. In systems such as speed control of DC motors, where the breakdown of machine restrictions causes serious injury, the use of DMC controller is highly suggested [5].

DMC controller implements the classic strategy of predictive controllers. Classical MPC strategy seeks to select the best possible set of control signals, within predetermined horizon, making explicit use of controlled process model. This application of DMC use the DC motor step response model to predict speed of the DC motor.

Another important part of classical MPC strategy is the implementation of moving horizon. The use of previous data indicates the current state of the process. In this application the past speed of DC motor and armature voltage are used in controller to increase its accuracy.

Beyond use of past data, moving horizon strategy limit the application of control signal proposed. This limitation occurs in order to update the data for increased accuracy. Repetition of steps mentioned at each sampling instant results in an increase in computational cost and an increase in controller accuracy.

Moving horizon strategy is completed by updating the data and proposing a new set of control signals at each sampling instant. Great care must be taken to enable the controller to perform all operations within the sampling period.

Fig. 2 illustrates the configuration of MPC algorithm. Parts of controller are illustrated, clearing the DMC strategy applied.

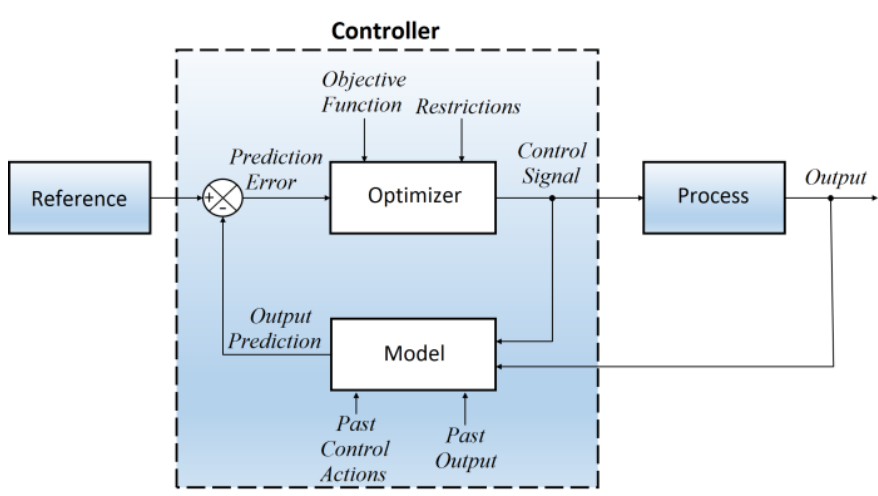

Figure 2. MPC controller block diagram

Restrictions of peak current Ip [A] and nominal armature voltage $\mathrm{VN}[\mathrm{V}]$ aimed at ensuring proper functioning of $\mathrm{DC}$ motor. The non-compliance of these restrictions cause bad engine operation and may cause irreversible damage to the machine. Insertion of these restrictions in DMC control law enables the controller to safely operate, close to the limits of machine, providing improved speed control performance.

The parameters of DMC controller to be optimized are: Prediction horizon R, Control horizon L and damping rate of reference signal $\alpha$

\section{Controllers Optimization}

Controllers' optimization aims, by choosing of values to controller's optimizable variables, minimize error between output of controlled system and proposed reference [6]. Deterministic, heuristic hybrid optimization methods are implemented to DMC controller applied to speed control of DC motor in order to compare them and establish which 
method provides best results. The best optimization method will provide the controller with less transient period and greater stability in steady state.

The deterministic method to be applied will be the QuasiNewton (QN) method, the heuristic method will be the Genetic Algorithm (GA). The hybrid method will combine the capacity to cover wide range of values from GA and accuracy of QN [9].

Given the fact that optimization methods seek to minimize defined fitness function, having no deep knowledge of system that pretends optimize, such optimization methods may suggest configurations that will bring damage to DC motor. Seeking to prevent that optimization methods propose gains to controllers that will bring damage to DC motor some penalties have been imposed to fitness function. These penalties are defined by maximum limits for armature voltage and current to the DC motor to be controlled.

The evaluation function to be used by all controllers' optimizators is the Integral of Absolute Error (IAE), calculated basing on error between speed reference and speed developed by DC motor IAE $\omega$. Taking penalties and evaluation function cited the fitness function is given by:

$$
f(x)=I A E \omega \cdot(\kappa+v)
$$

where: $\kappa=I a_{\max }-I_{p}$ and $v=V a_{\max }-V_{N}$, to $\kappa>0$ and $v>0$

The results given by (3) qualifies the controllers optimized. The best optimization method delivery the process with less error between proposed reference and speed developed by DC motor. The penalties have great influence, taking care of safety operation of DC motor. At end, the best optimization method delivery the process with minor errors, i.e., minor fitness function value.

\section{RESULTS}

\section{A. Modeling and simulation of direct current motors}

To perform the simulation, real parameters obtained from a commercial DC motor was used. These parameters are shown in Tab. I.

Table I

DC MOTOR PARAMETERS

\begin{tabular}{|c|c|}
\hline$J=0.032000167 \mathrm{Kgm}^{2}$ & $L_{a}=0.027089 \mathrm{H}$ \\
\hline$B=0.0022069 \mathrm{Nms} / \mathrm{rad}$ & $R_{a}=6.898 \Omega$ \\
\hline$K_{t}=1.073 \mathrm{Nm} / \mathrm{A}$ & $K_{b}=1.073 \mathrm{~V} / \mathrm{rad} / \mathrm{s}$ \\
\hline$V_{a}=230.0 \mathrm{~V}$ & $I_{p}=33.38 \mathrm{~A}$ \\
\hline
\end{tabular}

Applying the DC motor parameters obtained in (1), becomes possible to verify that the system has two real and distinct poles. Then, it is expected that the system response to input of step type is of over-dumped type as illustrated in Fig. 3.

The Fig. 3 present the speed developed by DC motor referred throughout experiment, being the input of the unit step type. The existing variation in instant $\mathrm{t}=10 \mathrm{~s}$ is caused due to load torque insert with numerical value of $2 \%$ of system reference value, being equal to $\mathrm{TL}=0.02 \mathrm{~N} \cdot \mathrm{m}$ for reference of $1.0 \mathrm{rad} / \mathrm{s}$.

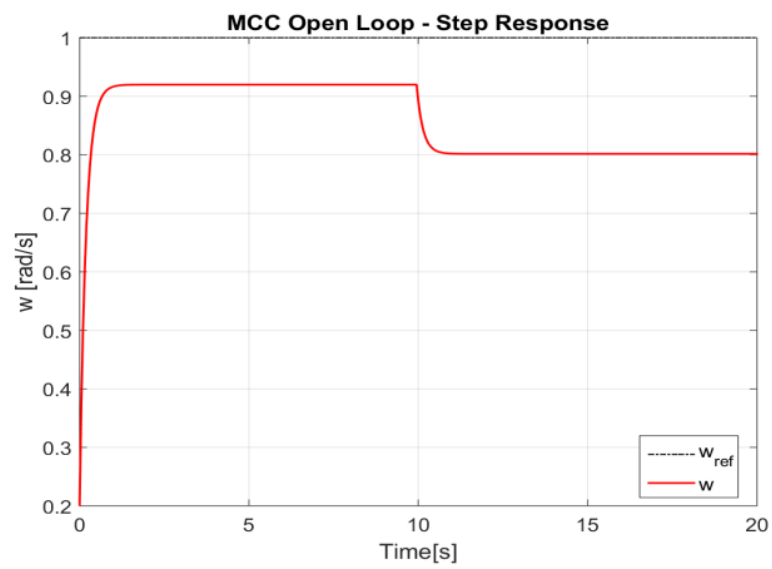

Figure 3. Step response of DC motor

\section{B. Controllers Optimization}

The optimizable parameters of DMC controller, R, L and $\alpha$, should respect the restrictions of MPC controllers, being 1) prediction horizon R: positive integer value, less than or equal to model horizon $\mathrm{N}$; 2) control horizon $\mathrm{L}$ : positive integer value, less than or equal to prediction horizon $\mathrm{R}$ and being 3 ) damping rate reference $\alpha$ : real value between 0 and 1 .

These restrictions are related to the optimization process, representing constructive restrictions of control technique and being not related to the highlighted restrictions in the system to be controlled.

The fitness function presented in (3) is implemented for all optimization methods. Taking the parameters of the commercial DC motor presented in Tab. I the restrictions are defined as $V_{N}=230.0 \mathrm{~V}$ to armature voltage and $I_{p}=33.38 \mathrm{~A}$ to armature current.

\section{Study Case 1: Deterministic Optimization of DMC}

Using the deterministic method, the optimization of DMC controller was realized starting from initial stochastic parameters. Fig. 4 present values of speed, armature current and armature voltage developed by DC motor controlled by DMC with parameters values obtained by Quasi-Newton method. It considered the set-point speed at $100 \mathrm{rad} / \mathrm{s}$ and inserted load of $2.0 \mathrm{~N} \cdot \mathrm{m}$ applied at time $\mathrm{t}=10 \mathrm{~s}$.

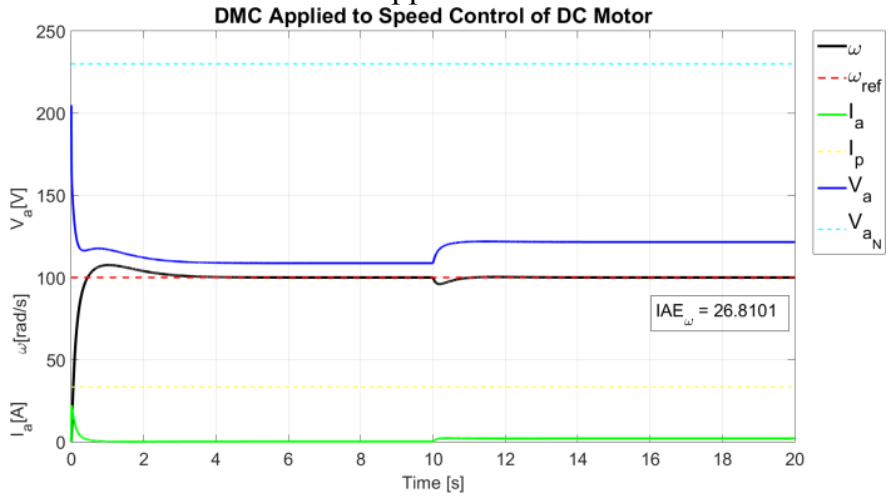

Figure 4. DC motor controlled by DMC tuned by deterministic method ( $\omega r$ f $=100 \mathrm{rad} / \mathrm{s}$ ). 
Analyzing Fig. 4 is noted higher values for armature voltage and armature current in early moments of the experiment, DC motor starting. Is noted also that with the stabilization of speed developed by DC motor in speed reference both the armature voltage and the armature current remain stable. In instant $t=$ $10 \mathrm{~s}$ the insertion of load with value $2.0 \mathrm{~N}$ causes disturbance to system slowing DC motor speed developed and making it necessary the increase of armature voltage and armature current values to that the speed developed reaches reference speed. Again, with stabilization of DC motor speed in speed reference both the armature voltage as the armature current remains stable. Fig. 4 yet present the integral of absolute error between reference speed and speed developed by DC motor being IAE $\omega=26.8101$.

Fig. 5 present values of speed, armature current and armature voltage developed by DC motor controlled by DMC with parameters values obtained by Quasi-Newton method. It considered the set-point speed at $50 \mathrm{rad} / \mathrm{s}$ and inserted load of $1.0 \mathrm{~N} \cdot \mathrm{m}$ applied at time $\mathrm{t}=10 \mathrm{~s}$.

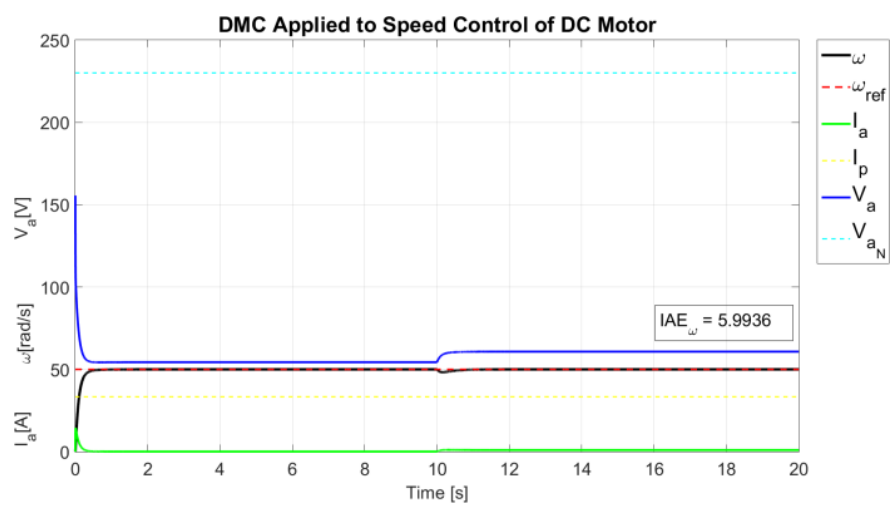

Figure 5. DC motor controlled by DMC tuned by deterministic method ( $\omega$ ref $=50 \mathrm{rad} / \mathrm{s}$ ).

Analyzing Fig. 5 is noted similarity to Fig. 4. Is noted higher values for armature voltage and armature current in early moments of the experiment and stabilization of these values with the stabilization of motor speed. Is noted the perturbation of system due to insertion of load with value of $1.0 \mathrm{~N}$ at time $\mathrm{t}=10 \mathrm{~s}$, similar to the previous experiment, it is evident the increase of armature voltage and armature current values aiming to return and the stabilization of speed developed by DC motor to reference speed. Fig. 5 yet present the integral of absolute error between reference speed and speed developed by DC motor being IAE $\omega=5.9936$.

Tab. II presents the total error found during the experiments and the optimal values for parameters of DMC controller obtained using the deterministic Quasi-Newton method.

Table II

RESULTS OF DMC CONTROLLER OPTIMIZED BY QUASI-NEWTON METHOD

\begin{tabular}{|c|c|c|c|c|}
\hline$\omega_{\text {ref }}$ & $f\left(x^{*}\right)$ & $R$ & $L$ & $\alpha$ \\
\hline 100 & 26.81014 & 15 & 9 & 0.00378 \\
\hline 50 & 5.99360 & 12 & 10 & 0.00366 \\
\hline
\end{tabular}

DMC controller tuning by deterministic (Quasi-Newton) method promote adequate performance of DC motor speed control. Note that with different set-point values different parameter are obtained. Another observation is that, with lower set-point values smaller fitness function values are obtained; this fact occurs, mainly, because the evaluation parameter is the Integral of Absolute Error of Speed IAE $\omega$ and with the reduction of set-point the error area is reduced in same percentage.

\section{Study Case 2: Heuristic Optimization of DMC}

Genetic Algorithm (GA) was implemented with an initial population of 20 individuals. Mutation and crossover rates were defined from linear variation where the mutation rate is $30 \%$ in the initial generation and $90 \%$ in the final generation and the crossover rate of $90 \%$ in the initial generation and $30 \%$ in the final generation. Selection method used was the tournament. The maximum number of generations Gmax was set at 100 generations.

Fig. 6 present values of speed, armature current and armature voltage developed by DC motor controlled by DMC with parameters values obtained by Genetic Algorithm method. It considered the set-point speed at $100 \mathrm{rad} / \mathrm{s}$ and inserted load of $2.0 \mathrm{~N} \cdot \mathrm{m}$ applied at time $\mathrm{t}=10 \mathrm{~s}$.

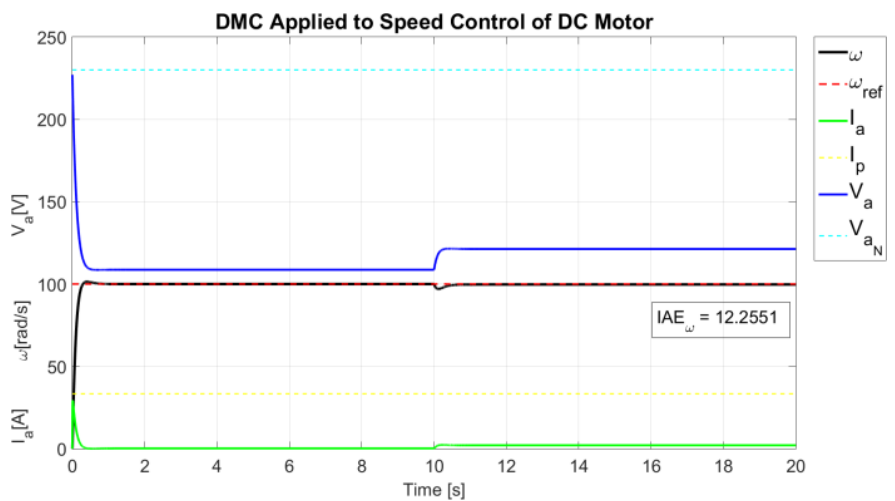

Figure 6. Speed of DC motor controlled by DMC tuned by heuristic method $(\omega \mathrm{ref}=100 \mathrm{rad} / \mathrm{s})$.

Analyzing Fig. 6 is noted higher values for armature voltage and armature current in early moments of the experiment, DC motor starting. Is noted also that with the stabilization of speed developed by DC motor in speed reference both the armature voltage and the armature current remain stable. In instant $t=$ $10 \mathrm{~s}$ the insertion of load with value $2.0 \mathrm{~N}$ causes disturbance to system slowing DC motor speed developed and making it necessary the increase of armature voltage and armature current values to that the speed developed reaches reference speed. Again, with stabilization of DC motor speed in speed reference both the armature voltage as the armature current remains stable. Fig. 6 yet present the integral of absolute error between reference speed and speed developed by DC motor being IAE $\omega=12.2551$.

Fig. 7 present values of speed, armature current and armature voltage developed by DC motor controlled by DMC with parameters values obtained by Genetic Algorithm method. It considered the set-point speed at $50 \mathrm{rad} / \mathrm{s}$ and inserted load of $1.0 \mathrm{~N} \cdot \mathrm{m}$ applied at time $\mathrm{t}=10 \mathrm{~s}$. 


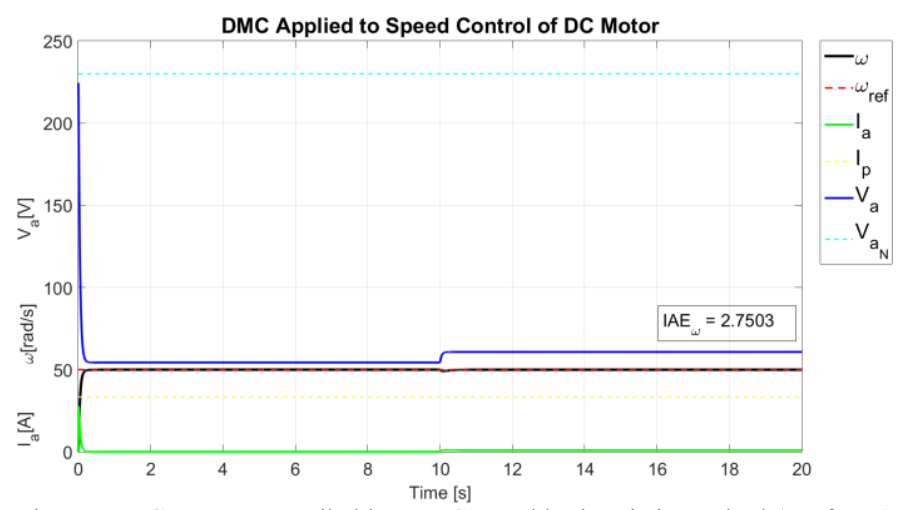

Figure 7. DC motor controlled by DMC tuned by heuristic method ( $\omega r e f=50$ $\mathrm{rad} / \mathrm{s})$.

Analyzing Fig. 7 is noted similarity to Fig. 6. Is noted higher values for armature voltage and armature current in early moments of the experiment and stabilization of these values with the stabilization of speed. Is noted the perturbation of system due to insertion of load with value of $1.0 \mathrm{~N}$ at time $\mathrm{t}$ $=10 \mathrm{~s}$, similar to the previous experiment, it is evident the increase of armature voltage and armature current values aiming to return and the stabilization of speed developed by DC motor to reference speed. Fig. 7 yet present the integral of absolute error between reference speed and speed developed by DC motor being IAE $\omega=2.7503$.

With the results obtained using the heuristic method, it is possible to observe the improvement in curves presented in relation to deterministic method. Tab. III presents the total error found during the experiments and the optimal values for parameters of DMC controller obtained using the heuristic Genetic Algorithm method.

Table III

Optimization of gains using the Heuristic Method.

\begin{tabular}{|c|c|c|c|c|}
\hline$\omega_{\text {ref }}$ & $f\left(x^{*}\right)$ & $R$ & $L$ & $\alpha$ \\
\hline 100 & 12.25510 & 9 & 5 & 0.00321 \\
\hline 50 & 2.75025 & 7 & 7 & 0.00299 \\
\hline
\end{tabular}

The optimization using the heuristic method allowed the improvement of the performance of DMC controller in response time and also in the annulment of the error in permanent regime. Fitness function values were reduced significantly in relation to values presented by implementation of deterministic method. Reduction of fitness function values represent better control of speed developed by DC motor.

\section{E. Study Case 3: Hybrid Optimization of DMC}

The implemented hybrid optimization initiates the search for optimized values realizing wide search within the set of possible solutions. To develop wide search hybrid optimization implements genetic algorithm. GA implemented at beginning of hybrid optimization repeats the characteristics of GA implemented isolated presented in III.D.

After wide search performed the hybrid optimization implemented seeks greater precision for solution presented until then. The hybrid optimization implements Quasi-Newton algorithm for refinement of the solution presented in the first step. The QN algorithm implemented in the hybrid optimization presents repeats the characteristics of $\mathrm{QN}$ implemented isolated presented in III.C.

Fig. 8 present values of speed, armature current and armature voltage developed by DC motor controlled by DMC with parameters values obtained by Hybrid method. It considered the set-point speed at $100 \mathrm{rad} / \mathrm{s}$ and inserted load of $2.0 \mathrm{~N} \cdot \mathrm{m}$ applied at time $\mathrm{t}=10 \mathrm{~s}$.

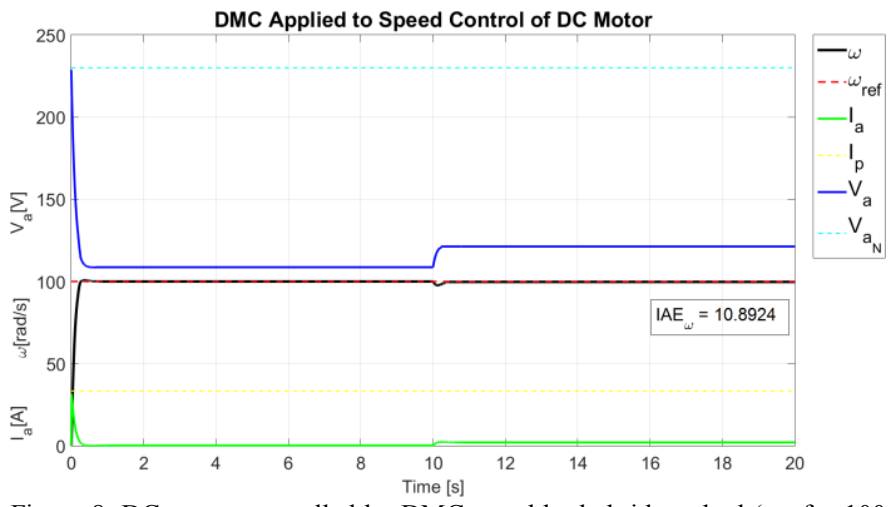

Figure 8. DC motor controlled by DMC tuned by hybrid method ( $\omega$ ref $=100$ $\mathrm{rad} / \mathrm{s})$.

Operation of DC motor controlled by DMC tuned by hybrid optimization method, Fig. 8, is similar to operation presented with optimization by heuristic method, Fig. 6. In speed developed by DC motor could be noted reduction of overshoot before speed stabilization. Note also, reduction in speed decrease caused by insertion of load with value $2.0 \mathrm{~N}$ at $\mathrm{t}=$ 10s. Characteristics evidenced in Fig. 8 illustrate reduction in integral of absolute error between reference speed and speed developed by DC motor, resulting in IAEw $=10.8909$.

Fig. 9 present values of speed, armature current and armature voltage developed by DC motor controlled by DMC with parameters values obtained by Hybrid method. It considered the set-point speed at $50 \mathrm{rad} / \mathrm{s}$ and inserted load of $1.0 \mathrm{~N} \cdot \mathrm{m}$ applied at time $\mathrm{t}=10 \mathrm{~s}$.

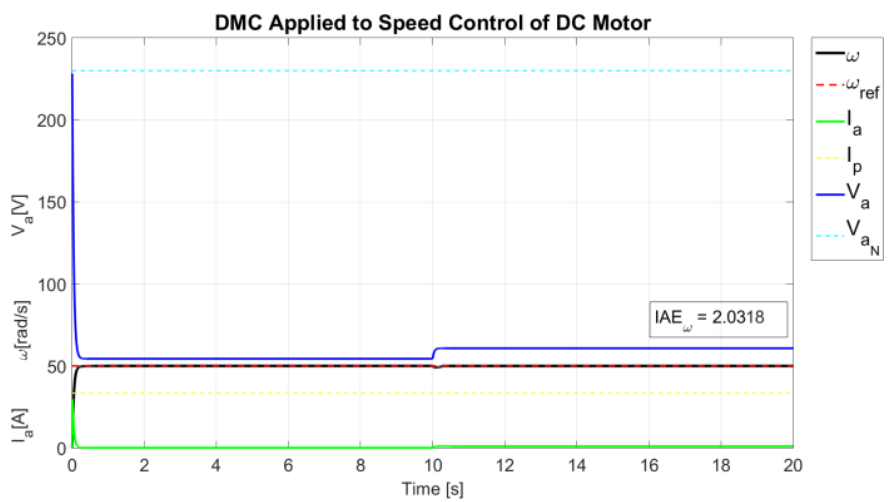

Figure 9 . DC motor controlled by DMC tuned by hybrid method ( $\omega r$ ref $=50$ $\mathrm{rad} / \mathrm{s})$.

Fig. 9 presenting operation of DC motor controlled by DMC tune by hybrid optimization method details operation 
similar to Fig. 8. Could be noted that even with a reduction of set-point the armature voltage and current still near of safety operation points at DC motor startup. Direct relation of armature voltage and current with speed developed by DC motor request high values of these parameters at DC motor startup. High armature voltage and current values at DC motor startup quickly drive speed to desired reference. Short period for high values of armature current and voltage guarantees absence of overshoots at speed developed by DC motor. Fig. 9 yet present the integral of absolute error between reference speed and speed developed by DC motor being IAE $\omega=2.031$.

With the results obtained using the hybrid method, it is possible to observe the improvement in curves presented in relation to heuristic method. Tab. IV presents the total error found during the experiments and the optimal values for parameters of DMC controller obtained using the heuristic Genetic Algorithm method.

Table IV

Optimization of gains using the Heuristic Method.

\begin{tabular}{|c|c|c|c|c|}
\hline$\omega_{\text {ref }}$ & $f\left(x^{*}\right)$ & $R$ & $L$ & $\alpha$ \\
\hline 100 & 10.89239 & 7 & 2 & 0.00191 \\
\hline 50 & 2.03181 & 7 & 1 & 0.00170 \\
\hline
\end{tabular}

Reduction presented in fitness function $f(x)$ values reflects the improvement of DMC controller with parameters tuned by hybrid optimization method in relation of tuning by other optimization methods. The improvement characterize minor error between speed reference and speed developed by DC motor.

\section{F. Comparison Between Optimization Methods}

Tab. V presents final values observed after execution of experiments where DC motor speed control was carried out by DMC controller tuned by deterministic method (QuasiNewton), by heuristic method (Genetic Algorithm) and by hybrid method (Genetic Algorithm/Quasi-Newton).

Table $\mathrm{V}$

DC Motor Parameters Final Value

\begin{tabular}{|clrll|}
\hline Optimizator & Set-point & \multicolumn{1}{c}{ IAE $\omega$} & Iamax & Vamax \\
\hline \multirow{2}{*}{ QN } & 100 & 26.8101 & 22.2045 & 204.8631 \\
& 50 & 5.9936 & 14.5460 & 155.5752 \\
\hline \multirow{2}{*}{ GA } & 100 & 12.2551 & 29.4314 & 227.0795 \\
& 50 & 2.7503 & 27.8424 & 224.4494 \\
\hline \multirow{2}{*}{ Hybrid } & 100 & 10.8923 & 32.6256 & 229.2125 \\
& 50 & 2.5033 & 29.7023 & 226.2568 \\
\hline
\end{tabular}

In Tab. V IAE $\omega$ values present the integral of absolute error between speed reference and speed developed by DC motor controlled by DMC tuned by different optimization methods. For smaller values of IAE $\omega$ smaller are errors occurred in the experiment consequently better is the proposed DMC parameters and better is the optimization method for the analyzed system.
For a speed set-point equal to $100 \mathrm{rad} / \mathrm{s}$ the controller tuned by hybrid method presents the lowest value of IAE $\omega$, characterizing itself as the best method for this system. The value of IAE $\omega$ presented by the system optimized by hybrid method is $11.12 \%$ smaller than the value presented when implemented heuristic method and $56.37 \%$ smaller when implemented deterministic method.

Similarly, for set-point equal to $50 \mathrm{rad} / \mathrm{s}$, the implementation of hybrid method presents better performance. The value of IAE $\omega$ presented by the system optimized by hybrid method is $8.98 \%$ smaller than the value presented when implemented heuristic method and 58.23\% smaller when implemented deterministic method.

Yet analyzing the data presented in Tab. V, for all the experiments, using parameters obtained from simulations, the armature voltage limits $\mathrm{Va}=\mathrm{VN}=230.0 \mathrm{~V}$ and armature current $\mathrm{Ia}=\mathrm{Ip}=33.38 \mathrm{~A}$ were respected.

In this article, the set of gains $\mathrm{R}, \mathrm{L}$ and $\alpha$ obtained through system optimization with speed set-point equal to $50 \mathrm{rad} / \mathrm{s}$ cannot be implemented when it is intended to operate the same plant with speed set-point equal to $100 \mathrm{rad} / \mathrm{s}$. To this operating characterizes, the DC motor restrictions, as peak current Ip = 33.38 and armature voltage $\mathrm{VN}=230.0$, are not respected, as shown in the figure below.

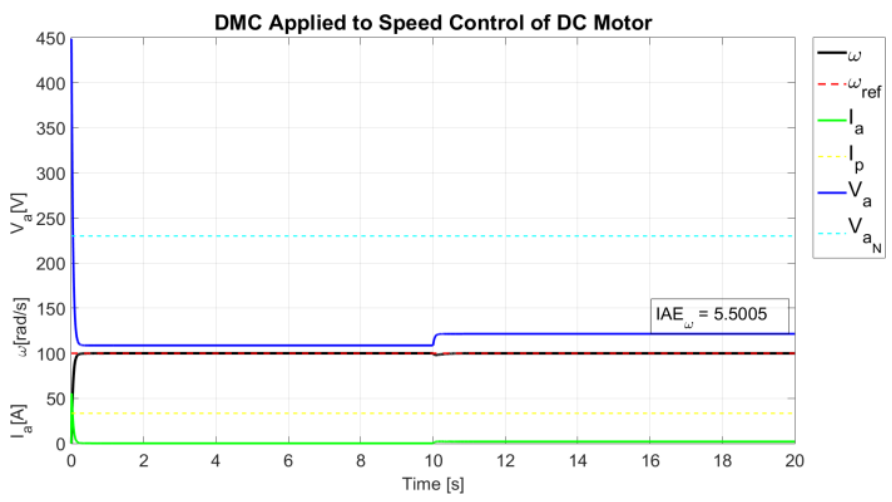

Figure 10. DC motor speed for system controlled by DMC tuned by $\mathrm{AG}$ for set-point $\omega \mathrm{ref}=50 \mathrm{rad} / \mathrm{s}$ applied for set-point $\omega r e f=100 \mathrm{rad} / \mathrm{s}$.

Yet analyzing Fig. 10, evidence the impossibility of applying the parameters obtained by optimization methods for the DMC controller with reference equal to $50 \mathrm{rad} / \mathrm{s}$ on the same controller with the reference equal to $100 \mathrm{rad} / \mathrm{s}$. It note peak current applied to the DC motor exceeding the threshold value, reaching Iamax $=55.6820 \mathrm{~A}$ and armature voltage applied to the DC motor exceeding the limit value, reaching Vamax $=448.8387 \mathrm{~V}$.

\section{CONCLUSION}

Held up the implementation of predictive DMC controller to speed control of DC motor, being employed to tune the controller, optimization techniques deterministic (QuasiNewton), heuristic (Genetic Algorithm) and hybrid (Genetic Algorithm/Quasi-Newton). The proposed optimized 
controllers were simulated for the same DC motor speed control in order to compare which optimization method obtain the most efficient controller, searching for reduction of the transient period and variations in continuous operation. To analyze the efficiency of the control developed was used as main criterion the Integral of Absolute Error of speed, presenting the existing error between the speed developed by DC motor and reference speed. Analysis of results shows a better performance of DMC controller optimized with hybrid method. Attentive to the fact that optimization for a given operating point does not guarantee safe operation of the controller in all parts of the system. Note that the tuning of the controller set-point of $50 \mathrm{rad} / \mathrm{s}$ afford gains that would outweigh the engine safety restrictions if the same gains are implemented in the same DMC controller, but seeking to reach set-point of $100 \mathrm{rad} / \mathrm{s}$. Finally, conclude that the implementation of DMC controller combined with the optimization parameters through the heuristic optimizer using genetic algorithm results in approach that shows promising results, enabling optimized DMC controller to be implemented in systems where search is control with high performance.

\section{ACKNOWLEDGMENT}

Authors thank the National Counsel of Technological and Scientific Development $(\mathrm{CNPq})$, the Research Support Foundation for the State of Goiás (FAPEG) and the Coordination for the Improvement of Higher Education Personnel (CAPES) for financial assistance to this research.

\section{REFERENCES}

[1] W. Goncalves da Silva, "Speed control of electric drives in the presence of load disturbances," Ph.D. dissertation, University of Newcastle upon Tyne, 1999.

[2] N. Nise, Control Systems Engineering. John Wiley \& Sons, 2007.

[3] C. R. Cutler and B. L. Ramaker, "Dynamic matrix control?? A computer control algorithm," in joint automatic control conference, no. 17, 1980, p. 72 .

[4] S. J. Qin and T. A. Badgwell, "A survey of industrial model predictive control technology," Control engineering practice, vol. 11, no. 7, pp. 733- 764, 2003.

[5] E. F. Camacho and C. B. Alba, Model predictive control. Springer Science \& Business Media, 2013.

[6] W. P. Calixto, T. M. Pereira, J. Mota, A. Alves, E. Domingues, J. Domingos, A. Coimbra, and B. Alvarenga, "Development of mathematical operator to heuristic optimization algorithms applied to problem of geoprospection, In Portuguese," TEMA (São Carlos), vol. 15 , no. 2, pp. 177-194, 2014.

[7] C. A. e. a. Ganzaroli, "Heuristic and deterministic strategies applied on a cascade pi controller tuning for speed control of a dc motor," IEEE Control Systems Magazine, 2015.

[8] S. Chapman, Electric machinery fundamentals. Tata McGraw-Hill Education, 2005.

[9] Vasant, Pandian M., Meta-Heuristics Optimization Algorithms in Engineering, Business, Economics, and Finance, IGI Global, 2012. 